\title{
Cytology and Neuron-Glial Apposition of Migrating Cerebellar Granule Cells in vitro
}

\author{
William A. Gregory, James C. Edmondson, Mary E. Hatten, and Carol A. Mason \\ Department of Pharmacology, New York University Medical Center, New York, New York 10016
}

In developing mammalian brain, many neurons migrate to their final position by moving in direct apposition to radially oriented glial cells. Glial-guided migration can be visualized in microcultures of mouse cerebellar cells by the combined use of cellular antigen markers and high resolution time-lapse video microscopy (Hatten et al., 1984; Edmondson and Hatten, 1987). Such studies have demonstrated the behavior of migrating cells and revealed a motile leading process on the migrating neuron that resembles an axonal growth cone and grows along extended glial fibers. To study the fine structural details of the migrating neuron and its neuron-glial apposition, we identified and monitored neurons in microcultures with video microscopy and examined the cytology and cellular contacts of the same cells with transmission electron microscopy.

The cytology of the soma and leading process of migrating cells closely matches that described for granule cells in intact brain (Rakic, 1971). Newly observed structures include the presence of longitudinally oriented microtubules extending from a basal body in the soma into the leading process, and microfilament-rich filopodia arising from the soma and leading process.

The most striking feature of actively migrating neurons is a specialized junction between the neuronal cell soma and apposing glial fibers. At this junction, here termed "interstitial density," the extracellular space is dilated to $20 \mathrm{~nm}$ and filamentous material in the intracellular cleft either spans the cleft or runs parallel to the cell membranes. Some interstitial fibrils are contiguous with, or are transmembranous extensions of, submembranous cytoskeletal elements that attach to microtubules. Interstitial junctions were not found between neurons that did not translocate in the observation period before fixation. Instead, stationary cells formed desmosomes (puncta and macula adhaerentia) at appositions with glial processes.

The migration of neurons along glial fibers is fundamental to mammalian brain development (Rakic, 1971, 1972, 1985; Rakic et al., 1974). Among cortical regions in the young brain, the

Received Apr. 27, 1987; revised July 29, 1987; accepted Aug. 25, 1987.

This work was supported by Grants NS-15429 and NS-21457. We thank our colleagues, Drs. Ekkhart Trenkner, Kon K. H. Liem, Michael V. L. Bennett, and David C. Spray for many helpful suggestions, and Mr. Jose Sanchez for technical assistance. Mr. Peter Peirce provided photographic assistance and Ms. Julia Cohen provided assistance with word processing.

Correspondence should be addressed to Mary E. Hatten or Carol A. Mason at their present address: Department of Pathology, College of Physicians and Surgeons, Columbia University, 630 West 168th St., New York, NY 10032.

Copyright (C) 1988 Society for Neuroscience $0270-6474 / 88 / 051728-11 \$ 02.00 / 0$ cerebellum provides a paradigm for glial-guided neuronal migration, that of the granule neurons along Bergmann glia. Cerebellar granule cells undergo their final cell division in the external granule layer, after which they become bipolar and elaborate processes that become the parallel fibers of the molecular layer (Ramon y Cajal, 1911). The granule cell soma then translocates perpendicular to the parallel fibers, migrating inward through the developing molecular layer in association with Bergmann glia (Rakic, 1971). This migration is disrupted in the neurological mutant weaver mouse (Rakic and Sidman, 1973; Sotelo and Changeaux, 1974), where neuron-glial interactions appear to be defective (Hatten et al., 1986).

The cellular and molecular mechanisms of neuron-glial interaction and migration can be studied with microculture systems (Trenkner and Sidman, 1977; Hatten and Liem, 1981; Hatten ct al., 1984, 1986; Trenkncr ct al., 1984; Hattcn, 1985; Mason et al., 1988). Recently, a detailed view of migration of cerebellar granule neurons along elongated astroglia has been observed in culture in real time by the use of high-resolution video-enhanced differential interference microscopy (Edmondson and Hatten, 1987).

To learn how cerebellar granule neurons move and what cellular structures are used during motility, we observed migrating granule neurons in vitro with video microscopy, then examined the cytology and appositions of the same cells with electron microscopy. As a comparison, we also studied cells that did not have a recent history of migration. A preliminary report of this work has been made (Gregory et al., 1986).

\section{Materials and Methods}

Cell cultures and video. Microculture dishes were prepared by punching an 8-mm-diameter hole in the center of culture dishes (Falcon 1006), and affixing an 18-mm-diameter No. 1 coverslip to the bottom of the dish with a 3:1 mixture of paraffin:Vaseline (Hatten and Liem, 1981). Monolayer microcultures of postnatal mouse cerebellum were prepared as described elsewhere (Hatten, 1985; Edmondson and Hatten, 1987). Briefly, purified cultures of astroglia derived from P3-4 C57BL/6J mice were grown for $24 \mathrm{hr}$ on a polylysine-treated $(100 \mu \mathrm{g} / \mathrm{ml})$ glass coverslip. Purified granule cells were then added, and after 1 additional day, cultures were observed. Cultures were maintained in a humidified incubator (Napco) at $35.5^{\circ} \mathrm{C}$ in $5 \% \mathrm{CO}_{2} / 95 \%$ air. For video-microscopic analysis, a glass coverslip was placed directly over the surface of the microculture and was sealed in place with silicon vacuum grease (Dow Corning). The microscope stage, objective, and condenser were enclosed in a heating chamber that was thermostatically controlled at $37^{\circ} \mathrm{C}$. Cultures were examined using a Zeiss IM inverted microscope equipped with differential interference optics (Edmondson and Hatten, 1987). The microscope was equipped with an analog contrast-enhancing video camera (Hamamatsu C1965-01). The video signal was passed through a time-date generator (MSI Video Systems), and was recorded on a timelapse optical disc recorder (Panasonic TQ 2025F), which sampled 1 frame $/ 4 \mathrm{sec}$, and was vicwed on a high-resolution video monitor (Sony PVM-122). 

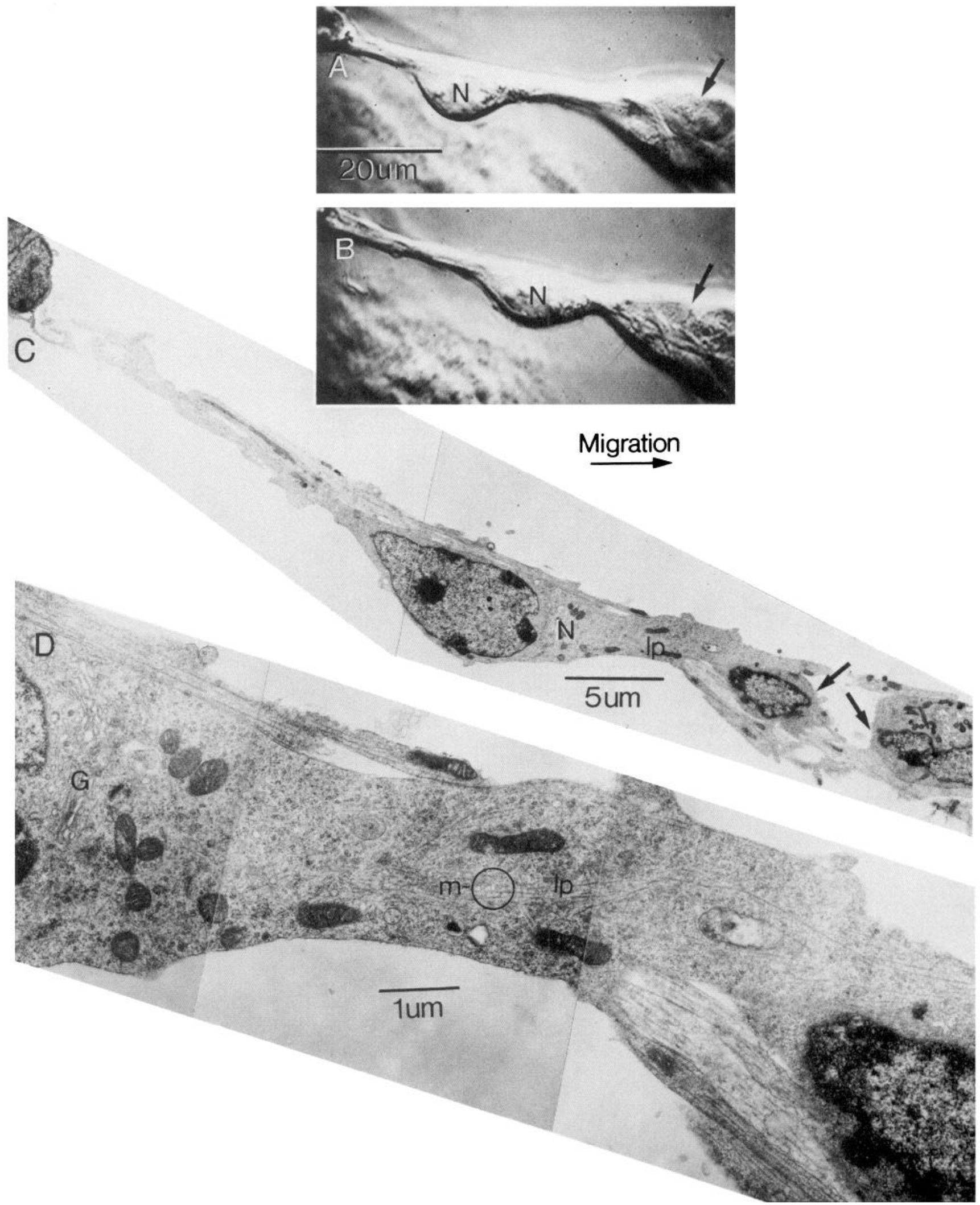

Figure 1. Electron micrographs of a migrating neuron that was fixed after observation with video microscopy. $A, B$, An elongated neuron $(N)$ is observed with video. In $B, 30 \mathrm{~min}$ after $A$, the neuronal soma is positioned to the right, indicating that migration toward a cluster of cells (arrows) has recently occurred. $C$, A low-magnification electron micrograph of the migrating neuron $(N)$ shows correspondence with the video representation in $B$. Arrows indicate cells in the cluster shown in video (arrows, $A, B)$. The leading process $(l p)$ is shown at higher magnification in $D$. $D$, The leading process $(l p)$ is a direct extension of the soma. The nucleus is present at far left, with adjoining Golgi $(G)$. The leading process contains primarily longitudinally oriented microtubules $(m)$, rosettes of ribosomes and mitochondria. The soma of this cell is shown in Figure 2. 

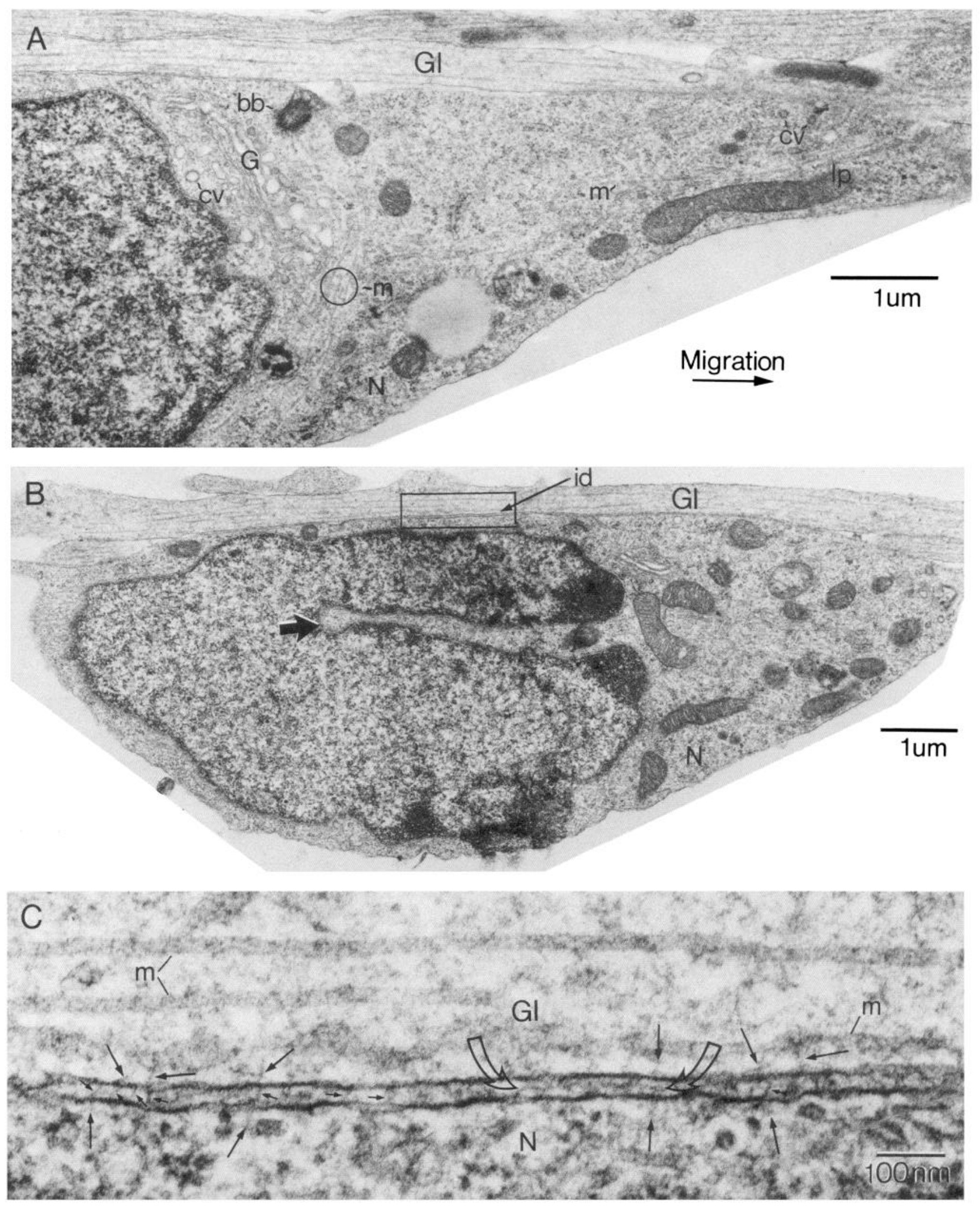

Figure 2. Electron micrographs of a neuron migrating along a glial process. The culture was fixed after observation of these cells with video microscopy. These are different sections through the same neuron shown in Figure 1. $A$, The rostral end of the soma contains Golgi $(G)$, basal body $(b b)$, coated vesicles $(c v)$, and ribosomes. Microtubules $(m)$ course around the nucleus and extend into the leading process $(l p)$. $B$, A different section through the same migrating neuron $(N)$ shows the characteristic caudal placement of the nucleus during migration, with a nuclear indentation on the forward surface (arrow). A region of pronounced interstitial density (id) is formed between the neuron and adjoining glial process (Gl) in the indicated rectangular region shown at higher magnification in $C$. $C$, Fibrillar interstitial material is evident between membranes of a migrating neuron $(N)$ and glial process $(G l)$. Fibrils span membranes (smaller solid arrows). Interstitial material is also located parallel to membranes (between 
Cultures were scanned for cells having the elongated (bipolar) shape typical of migrating neurons (Hatten et al., 1984; Trenkner et al., 1984; Edmondson and Hatten, 1987). A video record was made for $30-60$ min of selected cells, using a $63 \times$ planapochromat oil-immersion objective projected through a $20 \times$ eyepiece onto the video camera. Actively migrating neurons were defined as cells whose somata translocated at least one somatal length (approximately $10 \mu \mathrm{m}$ ) during the observation period. Cultures containing migrating neurons were then processed for electron microscopy. After video observation, the region was scribed with a false objective, and a $10 \times$ objective was used to record a low-magnification video record of the field showing the observed cell(s) and surrounding landmarks. In some cases, cells that failed to migrate in the observation period were also fixed in this manner.

Electron microscopy. The culture dish was flooded with fixative $(3.0 \%$ glutaraldehyde in $0.1 \mathrm{M}$ Sorensen's phosphate buffer, $\mathrm{pH} 7.2,22^{\circ} \mathrm{C}$ ). The upper coverslip was then gently removed, exposing the culture to fixative. Silicon grease remaining in the culture dish was removed using a cotton-tipped swab. The interval between final video observation and initiation of fixation was $2-5 \mathrm{~min}$. Cultures were fixed for $60 \mathrm{~min}$ at room temperature or overnight at $4^{\circ} \mathrm{C}$, then postfixed in osmium, stained en bloc in uranyl magnesium acetate, and dehydrated with ethanol. Cultures were infiltrated with an epoxy resin (Epox 812, Ernest F. Fullam). The microwell (lower) coverslip on which the cells had been grown was then separated from the culture dish by loosening the paraffin seal. Paraffin was scraped away from the coverslip with a razor blade, and the coverslip was polymerized with fresh resin against a plastic slide (Thomas Scientific, No. 6686V15)

The polymerized resin with attached glass coverslip was separated from the plastic slide and examined with a light microscope. The scribed circle and region observed with video were located, and an ink mark was placed with a felt marker (Sanford Sharpie extra-fine point, black) in the observed region on the plastic surface opposite the glass coverslip. The glass coverslip was then dissolved with concentrated stock hydrofluoric acid (10 min; Moore, 1975). The thin plastic film containing the embedded culture was then washed in water. The observed (ink-marked) region was cut out, the ink was erased with $70 \%$ ethanol, and the cutout portion was glued to a blank epoxy block using epoxy resin. The culture was oriented with substratum surface un so that sections would be taken parallel to the coverslip. Remaining portions of the embedded culture (regions not observed with video) were also saved for analysis.

The block containing the embedded culture was placed on a glass slide, and the position of the cells of interest was observed with a light microscope. The block face was trimmed to a suitable size for thinsectioning. A camera lucida drawing or pholograph was made showing the position and orientation of the observed cell on the trimmed block face so that the observed cell could be distinguished from other elongated neurons. Serial thin-sections were taken using a Sorvall MT-5000 ultramicrotome. Ribbons of silver sections were collected on Formvarcoated slot grids. Sections were poststained with uranyl acetate followed by lead citrate. Photographs taken from the video monitor and camera lucida drawings showing morphology, landmarks, and orientation and position of the observed cell on the block face were used to locate the video-observed cell during electron-microscopic examination. Every thin section containing the cell previously studied with video microscopy was examined with the electron microscope.

Parallel culture blocks were prepared from video cultures (regions not observed with video) and from cultures that were not subjected to video analysis. Cultures that were not first used for video microscopy were fixed by a gradual addition of fixative to the microwell shortly after the removal of the culture from the incubator. For better contrast and resolution, sections through parallel cultures were often collected on copper-mesh grids without Formvar.

Eight migrating cells recorded in real time with video microscopy were recovered for ultrastructural analysis. Two cells were recovered for ultrastructural analysis after it was verified that they were not migrating during the $30 \mathrm{~min}$ immediately prior to fixation. Hundreds of elongated neurons having a "migration posture" were examined in parallel cultures. The later cells came from numerous blocks from several cultures obtained from regions that were not subject to video exami- nation, and from several cultures that were fixed without video observation.

The details of structure and the quality of fixation showed no consistent variation between the cells that were subjected to video microscopy and those that were not. Preservation was good even for cultures subjected to video observation for 1-2 hr prior to the selection of a cell for video-electron-microscopic analysis. No changes in cellular behavior were observed with video after several hours of observation (unpublished observations and Edmondson and Hatten, 1987). Thus, the migration of neurons in this tissue culture system appears to be robust with respect to possible short-term effects of light-microscopic video examination on the structure, health of cells, or fixation.

\section{Results}

Cytology of migrating neurons and apposing glial processes

The leading process. The migrating neuron in this culture system has 3 primary parts: a leading process, a soma, and a trailing process (Figs. 1, $A-C ; 3, A-C$ ). A leading process extends from the soma in the direction of neuronal migration, shown at higher magnification in Figure $1 D$. The leading process contains a large number of ribosomes located primarily in rosettes, and contains numerous mitochondria (Figs. $1 D, 6 B$ ). Filopodia arise from the leading process and soma and contain microfilaments (Figs. $3 C ; 4, A-D ; 6 B)$. The motility of such filopodia has been observed with videomicroscopy (Fig. 4, $A, B$ ).

The tip of the leading process contains clumps of electron lucent vesicles (Fig. 4D). The tip is either expanded (Fig. 4, $A-$ $D$ ) or it ends bluntly (Fig. $6 B$ ). Coated vesicles are found within the cytoplasm of the leading process and coated omega figures are found on the plasma membrane of the leading process, the latter generally where it is apposed to other elements (Figs. $2 A$; $3 C, D$.

The soma. The rostral part of the soma tapers into the leading process (Fig. $2 A$; see also Fig. $1 C, D$ ). This region contains the same elements common in the leading process: ribosomes, mitochondria and microtubules, and can give rise to filopodia (Fig. $3 C$ ). In addition, the Golgi apparatus is located just ahead of the nucleus, with coated vesicles common in the surrounding region (Figs. $1 D ; 2 A ; 5, A, B)$. The rostral end of the nucleus of migrating granule cells has an indentation (Fig. $2 B$ ), confirming previous video observations (Edmondson and Hatten, 1987). The nucleus occupies the trailing portion of the soma. In this region the nucleus is surrounded by a thin rim of cytoplasm (Figs. $1 C, 2 B$ ). An axon-like trailing process with numerous longitudinally oriented microtubules extends caudally from the soma (not shown).

A basal body giving rise to a cilium is found in the rostral soma of both migrating cells and stationary cells with an elongated migration posture (Figs. $2 A ; 5, A, B$ ). Cilia and basal bodies are not seen in other regions of the neuron. The cilium of neurons with a migration posture, including neurons migrating at the time of fixation, is located primarily within the cell rather than protruding from it (Fig. $5 A$, from parallel culture). Cilia protrude from some spherical neurons located within clumps (not shown). Thus, the orientation of the cilium may vary with the activity of the neuron. Microtubules extend from the basal body to a submembranous position in the soma, and toward the micro-

$\longleftarrow$

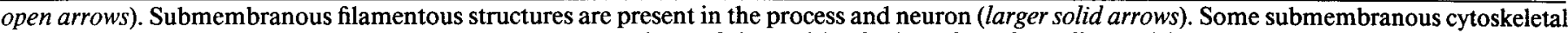

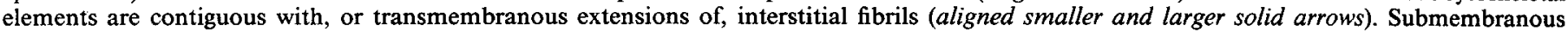
microtubules $(m)$ are present in the glial process. 

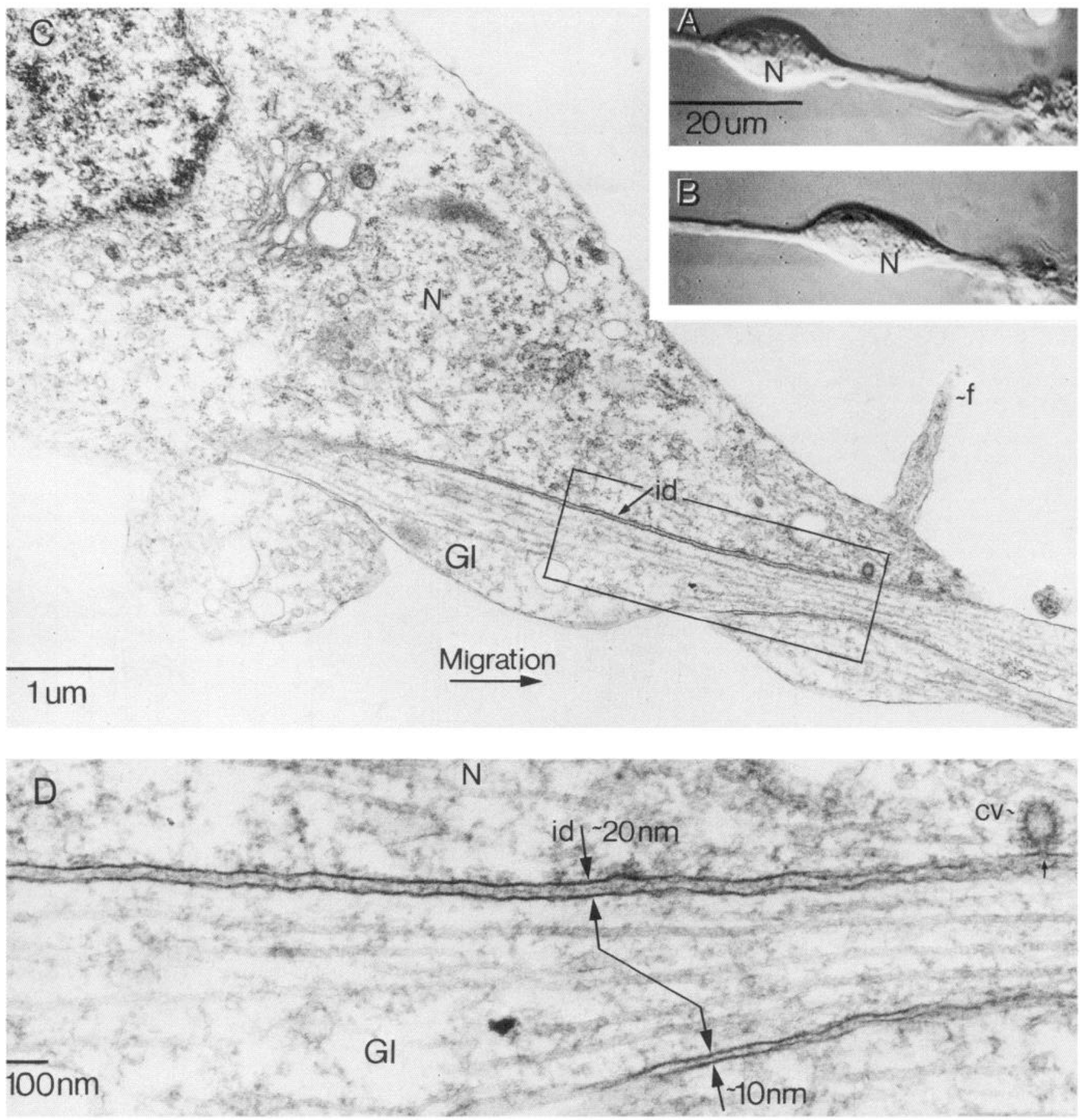

Figure 3. Electron micrographs of another neuron migrating along glia, fixed after observation with video microscopy. $A, B$, An elongated neuron $(N)$ is observed with video. In $B, 41 \mathrm{~min}$ after $A$, the neuronal soma is positioned to the right, indicative of recent migration. $C$, The rostral end of the neuronal soma $(N)$, extending into a leading process. A filopodium $(f)$ extends from the neuron. A region of interstitial density $(i d)$ is formed between the neuron $(N)$ and glial process $(G l)$ in the indicated rectangular region, shown at higher magnification in $D$. Serial section analysis showed intermediate filaments in the glial process (not shown). $D$, A region of interstitial density (id) contains interstitial material in dilated extracellular space (approximately 20 vs $10 \mathrm{~nm}$ elsewhere in these cultures). Both the neuron and process contain microtubules and demonstrate submembranous cytoskeletal material, as in Figure $2 \mathrm{C}$. A coated vesicle $(\mathrm{cv})$ is barely fused to the neuronal plasma membrane (small arrow) adjacent to the interstitial density region.

tubule rich leading process, while others appear to surround the nucleus (Figs. $2 A, 5 B$; see also Figs. $1 D, 5 A, 6 A$ ).

Glial processes. Migrating neurons maintain extensive contact with elongated astroglial processes, identified by the presence of intermediate filaments within these processes (Hatten et al., 1984, Mason et al., 1988). Intermediate filaments occur both as submembranous bundles (Figs. $5 B ; 6, A, C$ ) and as isolated filaments located in the cytoplasm (Figs. $4 E, 6 D$ ).

In some elongated glial cells, particularly those processes that are apposed to actively migrating neurons, intermediate filaments are not evident in every thin-section profile (e.g., Figs. $1-3$, filaments not shown), but isolated filaments can be located from serial-section analysis. Profiles containing sparse isolated 
intermediate filaments and numerous microtubules (Fig. $6 D$; see also Figs. 2-3) are considered to be glial, since intermediate filaments (neurofilaments) are not observed in processes which were clearly neuronal, and intermediate filaments are not generally observed in granule cells, the predominant neuronal form in these cultures (cf. Palay and Chan-Palay, 1974; Hatten et al., 1984). Intermediate filaments in elongated astroglia in culture consist, at least to a considerable extent, of glial filament protein (GFP), since GFP-immunoreactive elongated processes were observed with light microscopy adjoining elongated granule cells in parallel cultures (Hatten et al., 1984; Edmondson and Hatten, 1987). Dense bodies (Figs. 5B, 6C) and coated omega figures (Fig. $5 B$ ) are found within glial cytoplasm (see also Hatten et al., 1984). Filopodia also extend from elongated glial processes (Fig. 6C; cf. Mason et al., 1988).

\section{The cell-cell contact of migrating neurons}

Migrating neurons show extensive apposition with elongated glial processes. The membranes composing the neuron-glial apposition and adjoining submembranous cytoskeletons are specialized. The region of apposition between the somata of migrating neurons and adjoining glial processes has a dilated extracellular space ( 20 versus $10 \mathrm{~nm}$ elsewhere in the cultures) filled with a flocculent material (Figs. $2 C, 3 D$ ). As these junctional regions do not strictly resemble the classical named junction types of vertebrates, and biochemical, immunocytochemical, and physiological data are not yet available, we have adopted the generic term "interstitial density," a term also used for specializations of unknown significance between a variety of cell types in adult cerebellum (cf. Palay and Chan-Palay, 1974).

In some interstitial regions within interstitial junctions, material is oriented parallel to membranes, while in other regions fibrils span (are perpendicular to) membranes. In addition, submembranous cytoskeletal elements are contiguous with, or transmembranous extensions of, interstitial fibrils (Fig. 2C). Submembranous filamentous elements that might be microtubule-associated proteins (Hirokawa et al., 1985), or numerous other possible components of the membrane cytoskeleton, link the appositional membrane to microtubules that are commonly located in a submembranous position (Figs. $2 C, 3 D$ ). Submembranous cytoskeleton might be more obvious in the junctional region because the membrane is not extensively curved, as it is elsewhere in culture. Coated omega (endo/exocytotic) figures and coated vesicles, direct indications of membrane turnover, are found adjacent to interstitial densities of migrating cells (Fig. $3, C, D)$.

\section{Cytology of stationary neurons and apposing glial processes}

For comparison, we analyzed the cytology and cellular relations of cells that were not moving prior to fixation. Some stationary cells either lose or lack a leading process and have a spherical profile (Hatten et al., 1984; Trenkner et al., 1984; Edmondson and Hatten, 1987). An example of a spherical granule cell apposed to an elongated glial process from a parallel culture is shown in Figure $6 C$.

Two cells observed with video were examined with the electron microscope after verification that they were not migrating in the 30 min prior to fixation. Both of these cells have a migration posture, e.g., have an elongated profile with a tapered leading tip rather than a rounded profile (Hatten et al., 1984; Edmondson and Hatten, 1987). Cell A (not shown) is positioned part way down the length of an aggregate of elongated cellular processes. Cell $B$ is located at the end of an aggregate of elongated processes, oriented such that it may have migrated prior to video observation until it reached a clump of cells (Figs. $4, A, B$ ). The ultrastructural features of the apparent leading process, the soma and the apparent trailing process of these 2 stationary cells with a migration posture, show little difference from migrating cells. The position of the basal body, microtubules and cilia are the same as described above for migrating cells. In cell $\mathrm{B}$, the nucleus is located more rostrally and lacks the notch which is typical of migrating cells (Fig. $4 C$; cf. Fig. $2 B$ ), as previously observed with video time-lapsc microscopy of stationary cclls (Edmondson and Hatten, 1987). The nucleus of cell $A$ has the caudal placement of migrating cells. In addition, although the soma of cell $B$ is stationary, the video record shows cytoplasmic movement in the leading process-like neurite, and changes in the shape of the growth cone-like tip and filopodial movement (Fig. $4, A, B$,). Electron-microscopic examination reveals bundles of microfilaments in this neurite, extending into a filopodium (Fig. $4 D$ ). A clump of smooth vesicles is found at the end of this apparent leading process (Fig. 4D).

\section{The cell-cell contacts of stationary neurons}

Puncta adherentia rather than interstitial densities link neuronal somata with glia in two neurons that had elongated migration profiles but did not migrate in the 30-60 min observation period prior to fixation. Puncta and macula adherentia are never seen in cells with a history of recent migration. Desmosomes of various sizes (puncta and macula adherentia) also occur between the leading process or soma and the adjoining glial substrate (Figs. 5, $A, B ; 6 A$ ). In parallel cultures, where a fraction of neurons are presumed to be migrating at any one time (Edmondson and Hatten, 1987), puncta or macula adherentia are seen between some neurons and glia, while interstitial densities occur between other neurons and glia.

\section{Discussion}

Cytology of migrating neurons, with emphasis on the leading process

In general, the cytology of migrating granule neurons in our cultures closely resembles the description of migrating cells in vivo (Rakic, 1971, 1985), as well as previous studies of migration in vitro (Trenkner and Sidman, 1977; Hatten et al.,1984; Trenkner et al., 1984; Edmondson and Hatten, 1987). The leading process shares characteristics with neuronal growth cones, more so than is apparent in morphological studies in vivo (cf. Letourneau, 1981; Tosney and Wessells, 1983). The leading process and filopodia arising from it are motile (Edmondson and Hatten, 1987) and contain microfilaments that may be actin (Fine and Bray, 1971; Letourneau, 1981; Bray and Chapman, 1985). Microtubules, microfilaments, and submembranous cytoskeletal elements found both in migrating cells and growth cones presumably function in some fashion in force generation and as elements of structural support. In addition, leading processes resemble growth cones in that membranes are vesiculate. Even though this vesicular appearance may be an artifact of aldehyde fixation, as has been reported for the axonal growth cone (Rees and Reese, 1981; Cheng and Reese, 1985), the neuritic growth cone and leading process of the migrating cell share the same response to fixation.

Rakic (1971) reported that the morphology of the leading process of the monkey cerebellar granule cell migrating in situ did not resemble that of an axonal growth cone, and lacked filopodia. In contrast, migrating cortical neurons display filopodia and other growth cone-like features (Rakic, 1972). A re- 

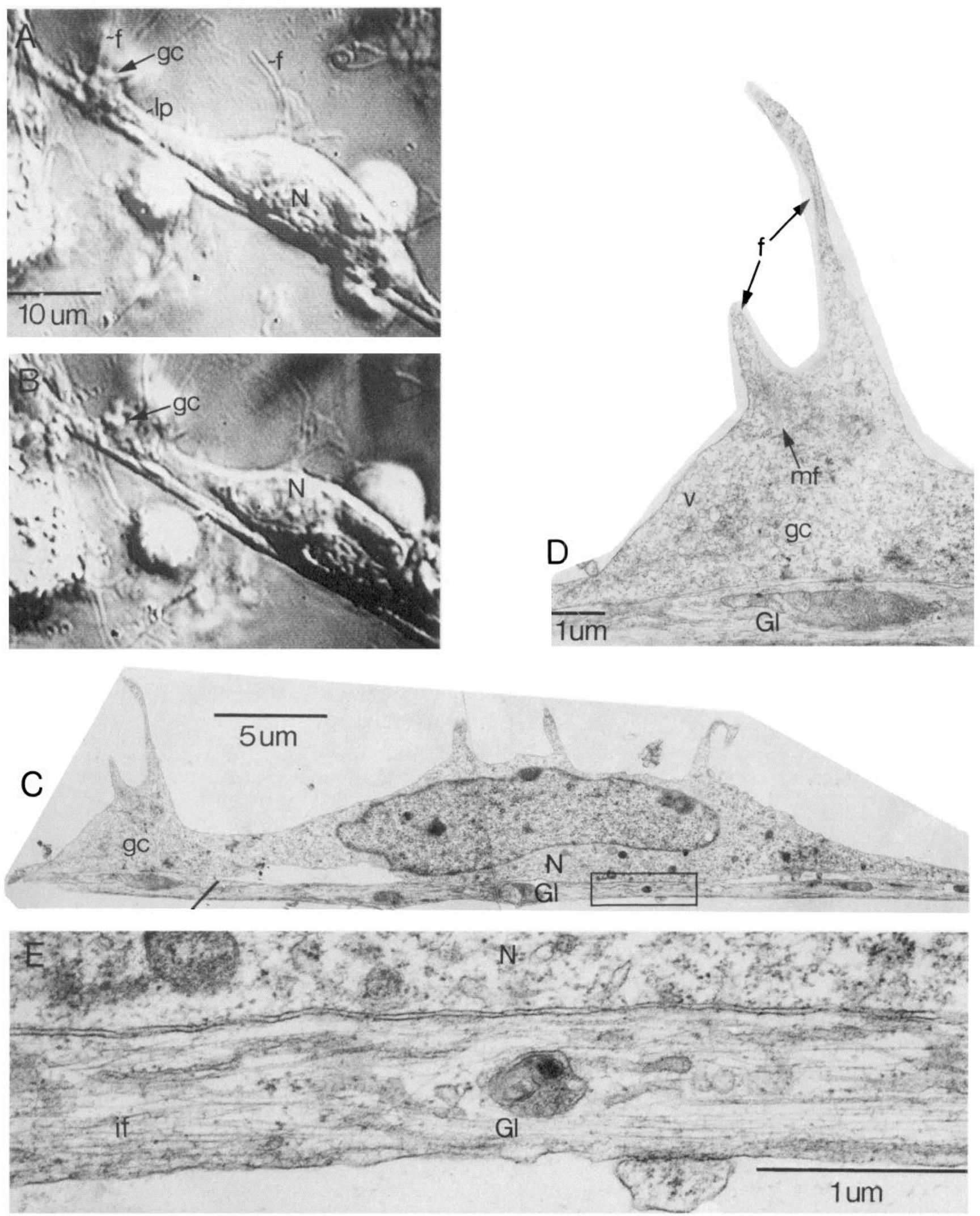

Figure 4. Electron micrograph of a video-observed neuron that did not migrate during the observation period, but had a migration (elongated) profile, and of a motile growth cone with filopodia at the terminal region of a leading process. $A, B$, The micrograph in $B$ was taken 15.4 min after that of $A$. The culture was fixed shortly thereafter. $A$ and $B$ were enlarged photographically to a higher magnification than that in the video micrographs in Figures $1, A, B$, and $3, A, B$. A growth cone-like $(g c)$ tip of the leading process, the leading process (lp), several filopodia $(f)$, and the apposition of the neuron with a process are visible. Movements of the growth cone were seen with video (compare $A$ and $B$ ). $C$, A lowmagnification electron micrograph shows the neuron $(N)$, glial process $(G l)$, and growth cone $(g c)$. Filopodia extend from the growth cone and from 
cent Golgi study of opossum indicates that some leading processes of granule cells end with growth cones and filopodia, while others apparently do not (O'Donoghue et al., 1987). These apparent differences between reports on the leading process in situ and ours, and the variation of leading process morphology within the cultures may be analogous to plasticity of growth cone form, depending on features of the local cellular microenvironment (Argiro et al., 1984). In addition, in intact tissue it is difficult to trace thin structures such as filopodia back to the parent growth cone unless the structure is selectively labeled (e.g., Bovelenta and Mason, 1987).

\section{Cell-cell contacts between migrating neurons and glia}

The soma of migrating cells form membrane specializations that we have termed interstitial densities. The leading process and neuronal soma of stationary neurons with a migration posture form puncta and macula adherentia rather than interstitial densities. Our data thus suggest a possible correlation between the type of junction formed by the neuron and the presence or absence of migration observed immediately prior to fixation. Interstitial densities may function in migration via roles in communication, motility, and/or adhesion. The presence of coated omega figures and coated vesicles adjacent to interstitial junctions of migrating cells, as well as near desmosomes, indicates turnover of junctional or nonjunctional membrane.

Specialized junctions have been noted between migrating neurons and radial glia in ncocortex, but details were not resolved (Rakic, 1972). The variation in maturation of adjacent regions of cerebellar development (e.g., Wassef and Sotelo, 1984), the saltatory nature of migratory movement (Trenkner et al., 1984; Edmondson and Hatten, 1987), and the fixation difficulties common to developing brain have probably contributed to the absence in other reports of junctions between migrating neurons and apposing glia.

A recent freeze-fracture study of developing rat cerebellum demonstrates regional and temporal differences in intramembranous particle density (Garcia-Segura and Rakic, 1985). Particle density was highest in the somatic region of the migrating neuron, lower in the leading process, still lower in the trailing process and parallel fibers, and very low in the growth cones of parallel fibers. Garcia-Segura and Rakic (1985) postulated that membrane is added to the leading process, as in growth cones, accounting for the difference in values for soma and leading process. In freeze fracture it would be impossible to distinguish cells which were actively migrating immediately prior to fixation, as we have done, from others with an elongated migration posture which, based on our studies, may not actually be moving. Although the functions of these intramembranous particles are unknown, the high intramembranous particle density reported for migrating somata could be another representation of the interstitial densities that we observed with transmission electron microscopy.

The classification of interstitial densities as junctions is perhaps a slight extension of the meaning of the term "junction" as applied to the more well-characterized categories of inter- cellular junction. It should be remembered, however, that the comparison of "traditional" forms of junctions from populations in fixed material, without use of video microscopy or other data relating to turnover, makes recognition and assessment of dynamic characteristics difficult. Moreover, the term "interstitial density" is not meant to imply any necessary functional or biochemical homology between the various membrane specializations having a similar morphological appearance (cf. Palay and Chan-Palay, 1974). Nonetheless, the present data showing interstitial densities between migrating cells and glia indicate that these are junctions in a functional and dynamic sense, i.e., specialized regional accumulations of membrane proteins between adjoining cells, one of which is translocating.

\section{The cytoskeleton: relations with membrane specializations, basal body, cilium, and flopodia}

It is of interest that many longitudinally oriented microtubules are found in the leading process. A basal body connects with the microtubules coursing toward the leading process, with microtubules that at least partially encircle the nucleus, and with submembranous microtubules in the soma. Submembranous microtubules are proximal to and apparently linked to the plasma membrane at regions of interstitial densities by cytoskeletal elements of unknown composition and function. Microtubules and glial intermediate filaments also closely adjoin adhaerens junctions. The microtubular association with membranes and with interstitial junctions of migrating cells suggests a possible role for microtubules and associated proteins in force generation, junction morphogenesis and in determination of cell shape. Ciliated neurons have been reported in vivo in numerous parts of developing nervous systems, but the significance of neuronal cilia is unknown, and sensory function has been postulated (cf. Peters et al., 1976). Rakic (1972) noted the likely structural relationship of microtubules in the leading process with a centriole found in the soma of neurons migrating within neocortex. Premigratory mouse cerebellar granule cells in the external granule layer have protruding cilia (unpublished observation).

\section{The process of neuronal migration: summary and potential mechanisms}

These results implicate specialized intercellular junctions during neuronal migration. The existence of interstitial densities on the somata of migrating cells suggests dynamic characteristics for these junctions. The presence of coated omega figures adjoining interstitial densities of migrating neurons may be direct evidence for turnover.

The characterization of the migrating cell's cytology raises many questions for subsequent cell biological studies. One possible model for cellular movement might include a combination of force-generational (cytoskeletal) mechanisms, membrane addition, deletion and recycling, and cell surface membrane movements to move the neuronal soma and growth cone-like leading process forward along the glia, with the neuron leaving behind a trailing process which becomes an axon. Contact-mediated

$\leftarrow$

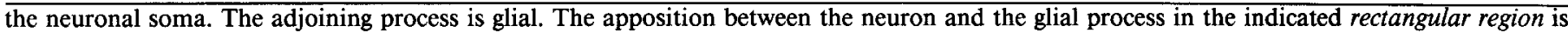

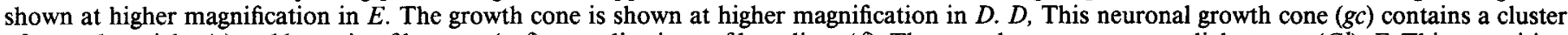

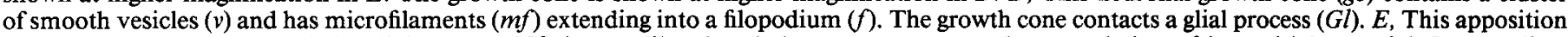

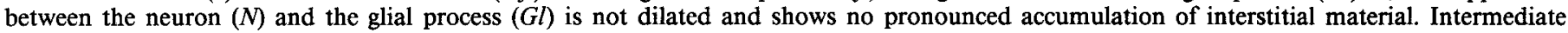
filaments (if) are numerous in the glial process. 

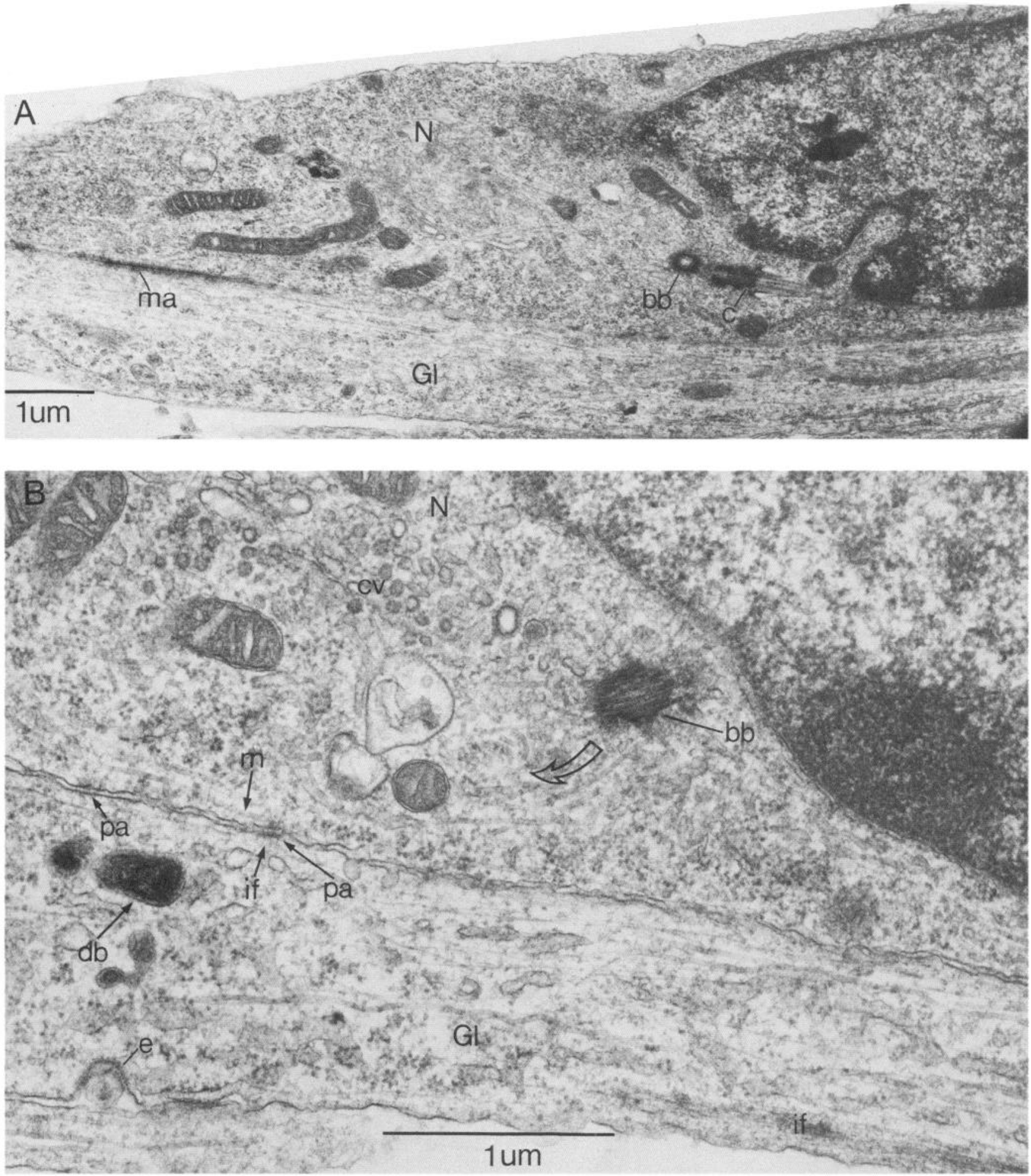

Figure 5. Electron micrographs of a neuron and glial cell from a parallel culture not observed with video microscopy. $A$, An elongated neuron $(N)$ with basal body $(b b)$ and cilium $(c)$ forms a maculum adhaerens $(m a)$ with a glial process $(G l)$. $B$, A different section through the same cells at higher magnification shows specializations of membranes and cytoskeleton. Microtubules ( $m$ and open arrow) course rostrally from the basal body $(b b)$ of the neuron $(N)$. Puncta adhaerentia $(p a)$ join the neuron with the glial cell $(G l)$. The glial cell contains intermediate filaments $(i f)$ and microtubules $(\mathrm{m})$. Submembranous cytoskeletal elements (a microtubule in the neuron, intermediate filaments in the glial cell) adjoin the puncta adhaerentia. Coated vesicles $(c v)$ are common in this region of the neuronal soma, which adjoins the Golgi. A coated endocytotic figure (e) is shown on the glial membrane. 

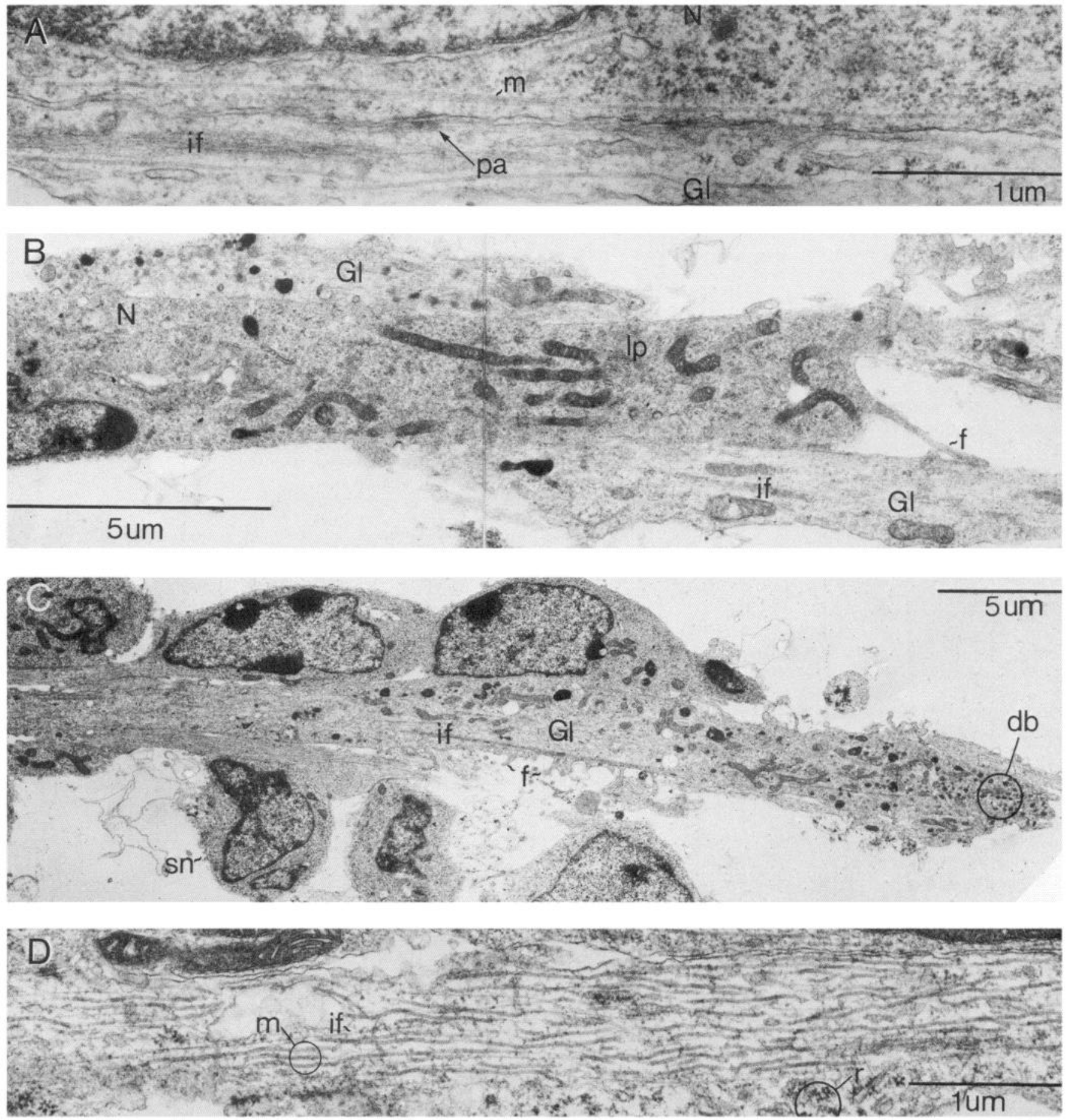

Figure 6. Electron micrographs of parallel cultures (cultures not observed with video). $A$, A neuronal soma $(N)$ is apposed to a glial $(G l)$ process. This neuron is elongated, with nucleus at upper left. An apparent leading process is present at far right (not shown). A submembranous microtubule $(\mathrm{m})$ is present in the neuron. The neuron forms a punctum adhaerens $(\mathrm{pa})$ with a glial process that contains intermediate filaments $(\mathrm{if})$. B, An apparent leading process $(l p)$ of a neuron $(N)$ is apposed to glial processes $(G l)$ containing intermediate filaments $(i f)$. A filopodium $(f)$ extends forward, contacting the glia. $C$, Several neurons are situated along an elongated glial process $(\mathrm{Gl})$. One neuron is spherical (sn); other studies (see text) indicate that spherical neurons are stationary. The glial process contains numerous dense bodies $(d b)$ that are probably lysosomes. The glial cell also contains intermediate filaments $(i f)$ and demonstrates short filopodia $(f)$. $D$, This glial process contains many microtubules $(m)$, isolated intermediate filaments (if), and ribosomes $(r)$.

interactions and timing mechanisms presumably regulate these phenomena (cf. Trenkner et al., 1984; Edmondson et al., 1988).

As the first correlated video time-lapse and ultrastructural study identified granule cells migrating along astroglial processes, this analysis demonstrates that the morphological details of cell-cell interactions can be directly related to cellular behavior in real time. Immuno-electron microscopy combined with video microscopy should prove useful in defining the molecular components of the types of junction observed in this study. Of particular interest will be whether junctional proteins, 
cell adhesion molecules, or other surface ligands such as astrotactin-antibodies which disrupt neuron-glial contacts in cerebellar microcultures (Edmondson et al., 1988)-are localized at these special cell-cell contacts. In addition, it will be important to address the relationship of cell surface interactional molecules to components of the cytoskeleton.

\section{References}

Argiro, V., M. B. Bunge, and M. I. Johnson (1984) Correlation between growth cone form and movement and their dependence on neuronal age. J. Neurosci. 4: 3051-3062.

Bray, D., and K. Chapman (1985) Analysis of microspike movements on the neuronal growth cone. J. Neurosci. 5: 3204-3213.

Bovolenta, P., and C. A. Mason (1987) Growth cone morphology varies with position in the developing mouse visual pathway from retina to first targets. J. Neurosci. 7: 1447-1460.

Cheng, T. P. O., and T. S. Reese (1985) Polarized compartmentalization of organelles in growth cones from developing optic tectum. J. Cell Biol. 101: 1473-1480.

Edmondson, J. C., and M. E. Hatten (1987) Glial-guided granule neuron migration in vitro: A high-resolution time-lapse video microscopic study. J. Neurosci. 7: 1928-1934.

Edmondson, J. C., R. K. H. Liem, J. E. Kuster, and M. E. Hatten (1988) Astrotactin: A novel neuronal cell surface antigen that mediates neuron-glial interactions in cerebellar microcultures. J. Cell Biol. 105: 505-517.

Fine, R. E., and D. Bray (1971) Actin in growing nerve cells. Nature 234: $115-118$.

Garcia-Segura, L. M., and P. Rakic (1985) Differential distribution of intramembranous particles in the plasmalemma of the migrating cerebellar granule cells. Dev. Brain Res. 23: 145-149.

Gregory, W. A., J. C. Edmondson, M. E. Hatten, and C. A. Mason (1986) Electron microscopic analysis of video-observed identified neurons migrating along glia in vitro. Soc. Neurosci. Abstr. 12: 369.

Hatten, M. E. (1985) Neuronal regulation of astroglial morphology and proliferation in vilir. J. Cell Biol 100: 384-396.

Hatten, M. E., and R. K. H. Liem (1981) Astroglia provide a template for the organization of cerebellar neurons in vitro. J. Cell Biol. 90: 622-630.

Hatten, M. E., R. K. H. Liem, and C. A. Mason (1984) Two forms of astroglia interact differently with cerebellar neurons in vitro. J. Cell Biol. 98: 193-204.

Hatten, M. E., R. K. H. Liem, and C. A. Mason (1986) Weaver mouse cerebellar granule neurons fail to migrate on wild-type astroglial processes in vitro. J. Neurosci. 6: 2676-2683.

Hirokawa, N., G. S. Bloom, and R. B. Vallee (1985) Cytoskeletal architecture and immunocytochemical localization of microtubuleassociated proteins in regions of axons associated with rapid axonal transport: The $\beta, \beta^{\prime}$-iminodipropionitrile-intoxicated axon as a model system. J. Cell Biol. 101: 227-239.
Letourneau, P. C. (1981) Immunocytochemical evidence for colocalization in neurite growth cones of actin and myosin and their relationship to cell-substratum adhesions. Dev. Biol. 85: 113-122.

Mason, C. A., J. C. Edmondson, and M. E. Hatten (1988) The extending astroglial process: Development of cell shape, the growing tip, and interactions with neurons. J. Neurosci. (in press).

Moorc, M. J. (1975) Removal of glass coverslip from cultures flat embedded in epoxy resins using hydrofluoric acid. J. Microsc. 104 : 205-207.

O'Donoghue, D. L., G. F. Martin, and J. S. King (1987) The timing of granule cell differentiation and mossy fiber morphogenesis in the opossum. Anat. Embryol. 175: 341-354.

Palay, S. L., and V. Chan-Palay (1974) Cerebellar Cortex: Cytology and Organization, Springer-Verlag, New York.

Peters, A., S. L. Palay, and H. deF. Webster (1976) The Fine Structure of the Nervous System: The Neurons and the Supporting Cells, Saunders, Philadelphia, PA.

Rakic, P. (1971) Neuron-glia relationship during granule cell migration in developing cerebellar cortex. J. Comp. Neurol. 141: 283-312.

Rakic, P. (1972) Mode of cell migration of the superficial layers of the fetal monkey neocortex. J. Comp. Neurol. 145: 61-84.

Rakic, P. (1985) Contact regulation of neuronal migration. In The Cell in Contact: Adhesions and Junctions as Morphogenetic Determinants, G. M. Edelman and J.-P. Thiery, eds., pp. 67-91, Neuroscience Research Foundation, New York.

Rakic, P., and R. L. Sidman (1973) Weaver mutant mouse cerebellum: Defective neuronal migration secondary to abnormality of Bergmann glia. Proc. Natl. Acad. Sci. USA 70: 240-244.

Rakic, P., L. J. Stensaas, E. P. Sayre, and R. L. Sidman (1974) Computer-aided three-dimensional reconstruction and quantitative analysis of cells from serial electron microscopic montages of foetal monkey brain. Nature 250: 31-34.

Ramon y Cajal, S. (1911) Histologie du Systeme Nerveux de l'Homme et des Vertebres, Maloine, Paris (reprinted by Consejo Superior de Investigaciones Cientificas, Madrid, 1955).

Rees, R. P., and T. S. Reese (1981) New structural features of freezesubstituted neuritic growth cones. Neuroscience 6: 247-254.

Solelo, C., and P. Changeaux (1974) Bergmann fibers and granule cell migration in the cerebellum of homozygous weaver mutant mouse. Brain Res. 77: 484-491.

Tosney, K. W., and N. K. Wessells (1983) Neuronal motility: The ultrastructure of veils and microspikes correlates with their motile activities. J. Cell Sci. 61: 389-411.

Trenkner, E., and R. Sidman (1977) Histogenesis of mouse cerebellum in microwell cultures: Cell reaggregation and migration, fiber and synapse formation. J. Cell Biol. 75: 915-940.

Trenkner, E., D. Smith, and N. Segil (1984) Is cerebellar granule cell migration regulated by an internal clock? J. Neurosci. 4: 2850-2855.

Wassef, M., and C. Sotelo (1984) Asynchrony in the cxprcssion of guanosine $3^{\prime}, 5^{\prime}$-phosphate-dependent protein kinase by clusters of Purkinje cells during perinatal development of rat cerebellum. Neuroscience $13: 1217-1241$. 